\title{
REFLEXÕES SOBRE OS VALORES DO MOVIMENTO SOFTWARE LIVRE NA CRIAÇÃO DE NOVOS MOVIMENTOS INFORMACIONAIS
}

\author{
Valdir Morigi \\ Dirce Maria Santin
}

\begin{abstract}
Resumo
Apresenta o software livre como movimento social, analisando sua identidade, seus adversários e seu modelo social. Reflete sobre os valores e as lutas do Movimento pela liberdade intelectual e sua contribuição na criação de novos movimentos informacionais, como o Cultura Livre, o Creative Commons e o Open Access. Disserta sobre a capacidade do software livre de mobilizar e articular comunidades a questionarem o processo de desenvolvimento das tecnologias de informação e comunicação, as formas de acesso à informação e a socialização do conhecimento, participando efetivamente e criando as condições necessárias à transformação dos valores e das instituições sociais na Sociedade da Informação.
\end{abstract}

\section{Palavras-Chave}

Movimento Software Livre; Cultura Livre; Creative Commons; Open Access; Liberdade Intelectual.

\section{INTRODUÇÃO}

$\mathrm{Na}$ sociedade atual todos os processos envolvem um conjunto cada vez maior e mais diversificado de informações. A Internet possibilitou transformações tanto nas realidades técnicas e econômicas quanto na produção e distribuição do conhecimen- to científico e do patrimônio cultural, configurando uma era informacional. Nesse cenário surgiram batalhas que mobilizam forças contrárias ao poderio legal dos sistemas de patentes e copyright em seu arranjo atual, ou seja, numa constituição que barra a inovação tecnológica, o acesso à 
informação, o uso livre do conhecimento e a socialização das criações humanas. Essas forças, indivíduos e grupos sociais do mundo todo, procuram potencializar a transformação tecnológica e cultural, participando efetivamente da criação e do uso do conhecimento e gerando condições para inclusão dos excluídos no processo de desenvolvimento da sociedade.

$\mathrm{O}$ artigo discute o software livre como um movimento social, seus valores em relação à liberdade intelectual e sua configuração no novo cenário de organização dos movimentos sociais: a Internet. Conforme a concepção de Touraine (1965), um movimento social se define a partir de sua identidade, de seu adversário e do modelo social proposto. Esses elementos permitem compreender como e onde o Movimento Software Livre (MSL) inscreve-se no debate pela liberdade intelectual e pelo uso livre do conhecimento.

O texto reflete sobre a influência do Movimento na criação de novos movimentos informacionais e sobre atuação e a forte referência aos termos do software livre em seus debates. Como exemplos para análise foram tomados os movimentos Cultura Livre, Creative Commons e Open Access, cujas lutas defendem o livre pensar, o direito de escolha, o acesso à informação e a socialização do conhecimento.

\section{O MOVIMENTO SOFTWARE LIVRE}

O termo "software livre" refere-se à liberdade conferida aos usuários para execução dos programas em qualquer propósito, para estudo do funcionamento e adaptação às necessidades específicas, para redistribuição de cópias a fim de estabelecer uma rede de relações solidária entre os cidadãos e para aperfeiçoamento do programa a partir de seu código-fonte. A palavra "livre" faz referência à liberdade e não ao preço, de modo que é possível pagar ou não para obter software livre (STALLMAN, 1993).

O software livre não é apenas uma forma alternativa de produção e distribuição de software, mas fundamenta-se em bases sólidas voltadas à transformação social, apoiado em valores como liberdade, igualdade, colaboração e socialização do conhecimento. No novo modelo social, o software livre tornou-se um movimento em defesa do uso livre e irrestrito das tecnologias de informação e comunicação (TICs) como processos desenvolvidos coletivamente e não apenas como instrumentos produzidos e impostos pelo capital e pelo mercado.

Os movimentos sociais são compreendidos na acepção de Castells (2000, p. 20) como: "[...] ações coletivas com um determinado propósito cujo resultado, tanto em caso de sucesso como de fracasso, transformam os valores e as instituições da 
sociedade". Nessa perspectiva, o software livre contribui e amplia a idéia de rede global proposta por Castells (1999), tornandoa real na medida em que congrega cidadãos dispostos a compartilhar seus conhecimentos em favor da sociedade, ou seja, na medida em que indivíduos e grupos sociais se apropriam das TICs e redefinem seus valores para geração de novos conhecimentos.

O software livre como um movimento social se insere no contexto histórico do surgimento da informática e da apropriação desta pelas grandes corporações. O Movimento teve origem com o lançamento do Manifesto GNU, escrito e publicado por Richard Stallman, em 1983, criando o conceito Free Software e dando início ao Projeto GNU (GNU's Not Unix!).

Stallman também criou Free Software Foundation (FSF), responsável por promover o desenvolvimento e o uso do software livre e pela representação jurídica dos programadores envolvidos no desenvolvimento de softwares do Projeto GNU, e a General Public Licence (GPL), uma licença que assegura a existência das liberdades fundamentais em versões de software reproduzidas ou melhoradas. A este tipo de licença, Stallman chamou copyleft (acordo com o autor), um conceito criado para enfrentar o copyright (direitos do autor) e recusar o constrangimento das inúmeras restrições impostas aos programadores para acesso ao código fonte dos programas (FSF, 1999).

O software livre como um movimento social, compreendido a partir da teoria proposta por Alain Touraine (1965), citado por Castells (2000), é definido de acordo com três princípios básicos: a identidade do movimento, seu adversário e sua visão ou seu modelo social, denominada por Castells como meta societal.

\subsection{A Identidade do Software Livre e seus Elementos}

O Manifesto GNU lançou o alicerce para a formação da identidade do MSL e sintetizou seus princípios fundamentais em sua autodefinição, traçando objetivos $\mathrm{e}$ anunciando em nome de quem se pronunciava. Afirmava a luta do Movimento por liberdade a todos os programadores e usuários de software, que passaram a reunirse em grupos na intenção de permitir que programas úteis fossem usados para fomentar uma comunidade de boa-vontade, disposta a lutar por liberdade em todo o conjunto da sociedade (STALLMAN, 1993).

Além de entender o Movimento em seus próprios termos, ou seja, naquilo que ele afirma ser no Manifesto GNU, importa compreendê-lo em suas práticas colaborativas no desenvolvimento de softwares e, especialmente, nas práticas discursivas dos debates promovidos pela comunidade 
internacional acerca das vantagens técnicas e políticas do uso de programas livres.

O Projeto GNU foi idealizado para favorecer o espírito solidário e cooperativo entre os programadores que prevalecia nos primórdios da informática. As práticas colaborativas adotadas pelas comunidades através da Internet conceberam um modelo baseado na solidariedade e na distribuição de resultados. Essas práticas passaram a exigir que os participantes estivessem dispostos à livre associação, em um esforço conjunto pela inovação tecnológica e pela democratização do acesso aos bens e serviços produzidos coletivamente.

O MSL fez da Internet seu principal meio de comunicação e, especialmente, de organização em comunidades. A Internet foi o impulso básico da organização e da adesão ao software livre, o que o instituiu como um movimento informacional de contestação à ordem social e econômica da Sociedade da Informação, e não apenas como uma filosofia contrária ao software proprietário.

Sem a intermediação dos meios de comunicação de massa e utilizando a Internet como tecnologia da liberdade, o MSL teve grande adesão por parte dos programadores e usuários de software. $\mathrm{O}$ engajamento desses agentes em comunidades e grupos sociais demonstrou a capacidade do Movimento de atrair pessoas dispostas a contribuir para a construção de um espíri- to solidário tão essencial quanto constituir um capital próprio.

A autonomia na organização das comunidades realça o elemento mais importante da identidade do MSL: a liberdade, desdobrada nas liberdades que sustentam a filosofia do Movimento e estimulam ações coletivas destinadas a transformar os valores e as instituições da sociedade. A freqüência com que o termo "liberdade" é repetido nos debates evidencia o caráter essencial e determinante desta como elemento imprescindível à identificação das comunidades e dos grupos sociais com o Movimento e demonstra que a principal vantagem do modelo é subjetiva: o software livre recupera a idéia de liberdade de acesso e uso do conhecimento, potencializando a noção de liberdade responsável, que faz com que indivíduos dos mais distantes países encontrem-se em fóruns e listas de discussão e compartilhem dos problemas e de suas soluções (MEFFE; GUALBERTO, 2005).

A identidade do MSL atribui, portanto, vantagens políticas sobre o modelo de produção do software proprietário, pois, independente de determinados programas serem melhores ou piores, a liberdade é um aspecto que o software proprietário nunca poderá superar. Caso o faça, cederá aos apelos do Movimento pela livre colaboração e pela disseminação dos resultados. 


\subsection{Os Adversários do Software Livre}

Para o MSL, a produção social do conhecimento não pode ser entendida como patrimônio de corporações ou estados, nem deve estar restrita ao exercício da autoridade e do poder. De acordo com Castells (2005), o software é a linguagem da era da informação e o controle tecnológico do software proprietário é equivalente à apropriação do alfabeto pelos escribas e sacerdotes no início da civilização.

Os adversários do Movimento atuam na subtração à liberdade intelectual dos cidadãos, criando mecanismos que excluem os não proprietários do processo de desenvolvimento tecnológico da sociedade. Para Kuhn e Stallman (2001), o software proprietário é um exercício de poder ao permitir que poucas pessoas tomem as decisões básicas sobre o software para todos, simplesmente negando suas liberdades.

Essa oposição à estrutura de poder do software proprietário ataca a conveniência comercial da obtenção de lucro pelas grandes corporações e as pressões do mercado, que provocam a inclusão de novas funcionalidades, cujo impacto é mínimo, e a obsolescência programada para garantir a venda de novas versões, além dos prazos de desenvolvimento e testes muito curtos para atender aos objetivos financeiros (HEXSEL, 2003).
A visão do poder ostensivo do modelo proprietário não se detém à comercialização do produto. Pereira (2004) alerta para a necessidade de perceber o software livre em uma perspectiva marxista, pois, na indústria de software, a verdadeira luta pelo controle do poder ocorre especialmente no processo de trabalho, ou seja, pela disponibilidade ou não do código-fonte. Assim, o código-fonte assume valor potencial, que é equivalente ao capital sob o poder das corporações, que controlam os meios e o conhecimento na geração de resultados.

Nessa concepção, o software livre difere da mercadoria, pois não tem valor de troca. O software livre é transferido a quem vai servir como valor de uso por meio do compartilhamento e não pela troca, embora seja possível comprá-lo. Assim, o MSL caracteriza-se como movimento revolucionário ao atacar os valores e as práticas meramente mercadológicas, enfatizando a necessidade de buscar a liberdade intelectual e preservá-la, por meio do copyleft (PEREIRA, 2004).

O software livre ataca a propriedade intelectual, cujos elementos, deformados no século $X X$, substituem gradualmente uma sociedade livre por uma sociedade de permissão. Essa "propriedade intelectual" reduz a muito pouco o conceito de propriedade criado para defender os autores e transfere aos serviços intermediários a prerrogativa legal do controle do uso da 
informação e do conhecimento (LESSIG, 2004).

O copyright e o registro de patentes se estabelecem como mecanismos desenvolvidos e aperfeiçoados para impor restrições de ordem ideológica, voltados aos interesses do capital e do mercado. Como alternativa, surge o copyleft, que propõe o uso consciente das criações humanas.

O Manifesto GNU discute os tipos de atos que a propriedade intelectual realmente autoriza uma pessoa a cometer e questiona a propriedade intelectual reconhecida pelo governo, criada por atos específicos de legislação para propósitos também específicos. Stallman (1993) defende que a situação dos programas hoje é bastante diferente daquela dos livros um século atrás. Na configuração atual do copyright, o usuário adquire apenas o direito de usar algo que é de propriedade de outro, de modo que precisa aceitar as limitações impostas pelo proprietário.

As possibilidades de flexibilização de direitos autorais e a liberdade intelectual são temas centrais do MSL. Essa oposição não faz do software livre um movimento favorável à pirataria, embora lute pelo uso irrestrito das criações humanas, especialmente na informática. As tentativas de qualificar o movimento como um grupo de hackers, no sentido atual da palavra, são contestáveis, pois a luta pela disponibilização de novos softwares sob a GPL de- monstra que o Movimento não aspira a cópia de programas proprietários, mas o desenvolvimento de alternativas estáveis pelas quais o usuário possa optar.

Enquanto o software livre avança frente às batalhas surgidas nos bastidores da Sociedade da Informação, também tenta restringir as tentativas técnicas criadas para reduzir a liberdade de compartilhar obras protegidas por direitos autorais. A versão 3 da GPL, em discussão, alerta para o risco criado pelo Digital Restrictions Management (DRM), um mecanismo criado para limitar, através de ferramentas instaladas no hardware e no software, o uso livre de trabalhos publicados (FSF, 2006).

Pela GPL3, nenhuma tecnologia pode invadir ilegalmente a privacidade e a liberdade dos usuários e nenhum mecanismo pode impedi-los de executar as obras licenciadas e exercer plenamente seus direitos de livre escolha e livre pensar. O surgimento do DRM alerta para os problemas provocados pela concentração de poder, ou seja, pelo desequilíbrio entre propriedade intelectual e liberdade intelectual. As conseqüências desse desequilíbrio provocaram, de uma forma menos visível e mais lenta, a criação de um novo modelo social para a Sociedade da Informação. 
2.3 Um modelo social para a Sociedade da Informação

A Sociedade da Informação criou novas formas de organização social, baseadas no uso das TICs. De acordo com Castells (2000, p.17):

Essa sociedade é caracterizada pela globalização das atividades econômicas decisivas do ponto de vista estratégico; por sua forma de organização em redes; pela flexibilidade e instabilidade do emprego e a individualização da mão-de-obra. Por uma cultura de virtualidade real construída a partir de um sistema de mídia onipresente, interligado e altamente diversificado. $\mathrm{E}$ pela transformação das bases materiais da vida - o tempo e o espaço mediante um espaço de fluxos e de um tempo intemporal como expressões das atividades e elites dominantes.

Nessa configuração, a Sociedade da Informação apresenta uma ordem social cujas forças transformam as condições existenciais da vida contemporânea, abalando estruturas sociais e criando novos modelos de organização e produção. Essa nova configuração passou a exigir dos indivíduos e grupos sociais estratégias de adaptação e ações específicas de reação frente às conseqüências excludentes dessa Sociedade.

Embora as reivindicações pela liberdade do MSL questionem de certa forma o modelo de produção capitalista, a visão ideológica do Movimento não é de todo socialista. Diante da amplitude alcançada pela contestação à nova ordem econômica global por diversos movimentos sociais, 0 MSL fortalece o debate sobre os aspectos políticos das TICs na construção de um novo modelo social e busca alternativas ao modelo vigente, que sacrifica muitos em detrimento de poucos.

A concepção do Movimento sobre o tipo de organização social ideal para a Sociedade da Informação supõe alguns elementos fundamentais, refletidos no conjunto de valores do movimento. A existência de um núcleo organizado no MSL, liderado pela FSF e por outras organizações representativas e o forte apelo à liberdade de organização compensam a diversidade de grupos e comunidades distribuídos no desenvolvimento de diferentes sistemas.

O modelo social proposto pelo MSL ataca a desigualdade social e intelectual como questão política, especialmente nas formas de organização, produção e distribuição de software, e busca o retorno à valorização do trabalho criativo do ser humano frente ao trabalho alienado e meramente mecânico, sem criatividade. Para Evangelista (2005, p.94):

[...] uma das marcas fundamentais do debate sobre software livre é a discussão entre o que é reconhecido como 'técnico' e o que é reconhecido como 'político'. [...] embora o software seja tido como algo técnico, a adoção de uma ou de outra tecnologia tem profundas conseqüências sociais que se refletem no imaginário sobre um ordenamento do mundo, principalmente relacionado à distinção ou não-distinção 
entre quem produz e quem só pode consumir.

Nessa perspectiva, as possibilidades técnicas dos programas livres não se sobrepõem ao forte apelo político do Movimento, que busca favorecer a reapropriação, por parte da sociedade, dos resultados de sua própria criatividade, gerando autonomia por parte das comunidades e grupos sociais. Essa autonomia tem provocado alterações sociais e culturais, especialmente na capacidade desses agentes de integrarem os debates políticos sobre as forças do mercado frente à inovação tecnológica.

\section{O MSL E OS NOVOS MOVIMENTOS INFORMACIONAIS}

Do software livre à cultura livre, o processo de construção de novos movimentos informacionais foi inspirado pelo MSL, que provocou o debate sobre o compartilhamento das criações humanas e opôs a solidariedade social à propriedade intelectual, provando ser capaz de propor alternativas eficientes por meio do livre intercâmbio de experiências.

A falta de equilíbrio entre os interesses públicos e privados e o tratamento indevido do direito de propriedade intelectual são obstáculos decisivos ao progresso material e à qualidade de vida na Sociedade da Informação. Segundo Saravia (2005), o conhecimento por natureza é livre, ainda que os sistemas legais herdados da Revolução Industrial, e já obsoletos, o converteram em um bem econômico escasso. Para Lemos (2004), não existe propriedade privada no campo da cultura, já que esta se constitui por intercruzamentos e mútuas influências.

A cultura livre, praticada além das fronteiras do MSL, defende o acesso livre a livros, músicas, filmes, softwares, sementes, tecnologias, artes e toda a investigação científica. Uma proposta recente, exemplo do desenvolvimento de novos movimentos informacionais, é o Cultura Livre, criado pelo professor americano Lawrence Lessig. A origem do Cultura Livre foi amplamente influenciada pelo MSL, que serviu de base à elaboração de muitas argumentações do Cultura Livre, incluindo a discussão sobre as conseqüências da substituição de uma sociedade livre por uma sociedade de permissão, determinada pelos argumentos contundentes da propriedade intelectual (LESSIG, 2004).

O Cultura Livre alerta para os problemas que a transformação tecnológica provoca quando seus usos são inquestionadamente aceitos como são atualmente e busca construir uma estrutura de autoorganização e participação na sociedade e na cultura, em oposição à estrutura proprietária. O Movimento procura agrupar os diversos movimentos informacionais, unindo-os por meio do Creative Commons e 
aproximando, numa existência complementar, aqueles que atuavam isoladamente, mas que já tinham por base o copyleft, originário do software livre (FREE CULTURE, 2006).

As tecnologias da informação e as mídias independentes criaram um novo paradigma para criação e uso do conhecimento, pelo qual qualquer um pode ser um artista, e qualquer um pode suceder o outro, baseado em seu mérito e não apenas em suas conexões com o mercado. Para Lessig (2004) a recusa ao "feudalismo digital" está fundamentada na liberdade intelectual, pela qual o cidadão pode construir sobre o que já foi criado, fazendo a criatividade e a inovação prosperarem. Nessa concepção, a cultura torna-se um caminho em dois sentidos, sobre participação, e não meramente sobre consumo.

O Cultura Livre criou formas alternativas de direito de propriedade adaptadas a nova função social da propriedade na Sociedade da Informação, com o lançamento do projeto Creative Commons, em 2001. O termo Creative Commons vem de res comune, conceito empregado pelo direito norte-americano para se referir aos bens considerados de todos, que não estão sujeitos ao controle de nenhuma entidade especifica. O Projeto disponibiliza opções flexíveis de licenças que garantem proteção e liberdade para artistas e autores e define um espectro de possibilidades entre copyright, de todos os direitos reservados, e domínio público, de todos os direitos livres.

Essas licenças protegem o conceito de autoria, permitindo que o autor estabeleça sob quais condições sua obra pode ser usada, sem ferir uma das principais conquistas da Internet: a livre circulação das idéias. O Creative Commons surgiu como alternativa para enfrentar as divergências do direito autoral frente ao avanço tecnológico. Para Stix (2003), o Creative Commons ultrapassou argumentos acadêmicos e, com base no modelo de software livre, traduziu palavras em ação, buscando soluções para o compartilhamento do trabalho criativo.

A influência do MSL sobre o Creative Commons não se limita aos valores fundamentais que regem a liberdade de uso do conhecimento. As licenças Creative Commons foram precedidas pela GNU Free Documentation License (GNU FDL), criada para licenciar a documentação do Projeto GNU. Shwingel (2006) afirma que apesar das evidências de derivar do software livre, o Creative Commons muda um pouco a tática de confronto com a lógica proprietária do copyright ao propor uma licença que busca um equilíbrio sustentado entre compromissos e moderação, utilizando o direito privado para criar bens públicos.

Stallman (2006) entende que as licenças Creative Commons são inaceitá- 
veis, pois nem todas são livres, e o público tende a ver o conjunto delas. Para ele, que já apoiou o Projeto em sua origem, o primeiro passo para pensar claramente sobre estas licenças seria discutí-las separadamente. $\mathrm{O}$ debate está em aberto, inclusive nos eventos do software livre. De qualquer forma, o Creative Commons adaptou as lições e o modelo do software para a área da cultura, atentando para suas peculiaridades. Ao oferecer aos artistas opções flexíveis sobre quais os direitos pretendem permitir que a sociedade usufrua, o Projeto foi assimilado rapidamente por artistas do mundo todo.

Outro movimento informacional que ganhou amplitude nos últimos anos é o Open Access. Instituído em 2001 pela Budapest Open Access Initiative ${ }^{1}$, o Movimento surgiu para modificar a rea-lidade da distribuição do saber científico e do patrimônio cultural, com base no acesso livre e universal ao conhecimento humano. $\mathrm{O}$ Open Access promove o uso livre dos meios de comunicação como mecanismos de divulgação do conhecimento e das instituições cientificas, dos financiadores, das bibliotecas, dos arquivos e museus do mundo todo.

\footnotetext{
1 Iniciativa e documento que fez a declaração de princípios, a definição da estratégia e a afirmação de empenho dos apoiadores do Movimento. Representa uma das primeiras reações da comunidade científica, resultado de uma reunião realizada em 2001, promovida pela Open Society Institute (OSI), para discutir a questão do acesso à literatura científica.
}

A Declaração de Berlin² 2003 define o acesso aberto como uma fonte extensa do conhecimento humano e do patrimônio cultural, validados pela comunidade científica. O Movimento surgiu como solução para enfrentar a crise da comunicação científica, gerada por barreiras legais e tecnológicas, aumentando a disseminação dos resultados das pesquisas e diminuindo os custos de acesso às publicações. O Open Access busca repropriar-se da comunicação científica e das TICs para disseminação livre na Internet da literatura acadêmica ou científica nos moldes do software livre, permitindo a leitura, o download, a cópia e a distribuição dos estudos consultados.

De acordo com Costa $(2006$, p.1) a fiIosofia aberta:

[...] refere-se ao movimento em direção ao uso de ferramentas, estratégias, metodologias e políticas que denotem um novo modelo de representar o processo de comunicação científica especialmente no que concerne à publicação, ao mesmo tempo em que serve de base para interpretá-lo. Esse novo modelo tem como fundamento a preocupação crescente com a disponibilidade, ao maior número possível de interessados, do conhecimento gerado como resultado tanto de pesquisas científicas (conhecimento científico) como da ação do homem na sociedade (herança cultural).

Para Aliprandi (2005), a mudança para o modelo Open Access modifica a disseminação do conhecimento nos seus as-

\footnotetext{
${ }^{2}$ Documento que compreende os resultados de reunião realizada primeiramente em 2003 e, anualmente, nos anos seguintes, em Berlim.
} 
petos tecnológicos, legais e financeiros, e as ferramentas, os normativos e meios de comunicação precisam ser compatíveis e igualmente de acesso aberto. Nesse sentido, a promoção do paradigma do acesso aberto pode oferecer o máximo benefício à ciência e à sociedade, promovendo uma rede sustentável, interativa e transparente. Além da infraestrutura de acesso, o Movimento também encoraja os pesquisadores a publicarem os resultados de seu trabalho nos repositórios abertos, como forma de lutar e contribuir para a continuidade do acesso livre às publicações eletrônicas, preservando os padrões de qualidade da validação pelos pares e da boa prática científica.

As perspectivas para o Open Access incluem vasta disponibilidade de estudos científicos on-line, sem barreiras de acesso e uso, sistemas transparentes e democráticos de recuperação da informação e novos mecanismos de avaliação e financiamento da ciência e dos pesquisadores. $O$ debate sobre o acesso aberto começa a ganhar espaço na discussão sobre as mudanças no processo de comunicação científica, dados os resultados das iniciativas concretizadas em repositórios de livre acesso. Assim, o Open Access reflete as mudanças tanto no ambiente da pesquisa quanto na produção e disseminação do patrimônio cultural, visando integrar soluções para socialização ampla e irrestrita do conhecimento.

\section{CONCLUSÕES}

O software livre como um movimento social tem a capacidade mobilizar e articular grupos sociais a questionarem o processo de desenvolvimento das tecnologias de informação e comunicação, as formas de acesso à informação e o uso livre do conhecimento, possibilitando a transformação das instituições sociais em torno dos seus valores.

O desenvolvimento da informática e o acesso dos cidadãos às tecnologias digitais por si só não garantem uma mudança qualitativa nas relações de poder engendradas pelo mundo capitalista. É necessária a viabilização de valores sociais que dêem sustentação e ampararem a filosofia e a identidade do MSL, estimulando práticas solidárias destinadas a transformar os valores e as instituições sociais. Ainda que os impulsos iniciais tenham acontecido através dos movimentos informacionais, muito precisa ser feito para tornar a informação e o conhecimento acessível e democrático, ultrapassando as barreiras tecnológicas ou ideológicas.

Um modelo social não se sustenta apenas com o desenvolvimento das ferramentas tecnológicas de seu acesso e uso. $\mathrm{O}$ componente subjetivo é elemento im- 
prescindível à identificação das comunidades e dos grupos sociais com o Movimento. Por isso, é necessário encorajar a discussão sobre a importância dos valores subjetivos na construção da cidadania, entre os quais a liberdade, como forma de potencializar a construção de redes de relações solidárias entre cidadãos de diferentes culturas, capazes de enfrentar problemas comuns e de participar do compartiIhamento das suas soluções.

$O$ direito de acesso informação e o uso livre do conhecimento são considerados direitos sociais básicos na construção da cidadania, pois o seu exercício pleno pressupõe o acesso às informações e aos saberes produzidos e distribuídos socialmente. Nessa perspectiva, a cidadania plena só será alcançada se a informação e o conhecimento forem acessíveis a todos, sem nenhum tipo de obstáculo, ou seja, sem a interferência dos direitos de propriedade tal e como estão determinados por regras que protegem as corporações controladoras do capital material e simbólico.

Acreditamos que a informação e o conhecimento não devem ser propriedade privada, pois o livre intercâmbio de experiências enriquece a cultura e o desenvolvimento tecnológico. Nosso conhecimento do mundo constitui-se de experiências vividas e das práticas culturais herdadas, pois somos o resultado do que lemos, vemos, ouvimos e vivenciamos em sociedade. A liberdade intelectual e os direitos de propriedade intelectual não devem ser opostos, mas complementares.

O MSL definitivamente provocou 0 debate sobre o livre intercâmbio de experiências na Sociedade da Informação. A identidade do Movimento e as lutas de oposição ao modelo proprietário vislumbram a criação de um modelo social baseado na liberdade intelectual e no uso livre do conhecimento para toda a sociedade, culminando na criação de novos movimentos informacionais, como o Cultura livre, o Creative Commons e o Open Access. Algumas vezes, esses movimentos aparecerão apenas como alternativas ao desenvolvimento tecnológico. Outras vezes, poderão configurar-se como caminhos autônomos e interdependentes, capazes de transformar os cidadãos e sua subjetividade, as instituições sociais e seus valores na Sociedade da Informação.

\section{REFERÊNCIAS}

ALIPRANDI, Simone. Open Access. In: ALIPRANDI, Simone (Org.). Compendio di libertà, informatica e cultura open. Roma: Primaora, 2005. p.151-154. Disponível em: $<$ www.copyleft-italia.it/compendio/aliprandicompendio.pdf>. Acesso em: 27 jan. 2007.

CASTELLS, Manuel. A sociedade em rede. 6.ed. São Paulo: Paz e Terra, 1999. Inovacion, liberdad y poder en la era de la informacion. Porto Alegre: Fórum Social Mundial, 29 jan. 2005. Disponível em: 
$<$ http://www.softwarelivre.org/news/3635>. Acesso em: 20 jan. 2005.

O poder da identidade. 5.ed.

São Paulo: Paz e Terra, 2000.

COSTA, Sely M. S. Filosofia aberta, modelos de negócios e agências de fomento: elementos essenciais a uma discussão sobre o acesso aberto à informação científica. Ciência da Informação, Brasília, DF, v.35, n.2, p.39-50, maio/ago. 2006. Disponível em:

$<$ http://www.ibict.br/cienciadainformacao/vi ewarticle.php?id=926>. Acesso em: 20 mar. 2007.

EVANGELISTA, Rafael de Almeida.

Política e linguagem nos debates sobre $o$ software livre. 2005. Dissertação (Mestrado em Lingüística) - Universidade Estadual de Campinas, Campinas, 2005. Disponível em:

<http://libdigi.unicamp.br/document/?code= vtls000349663>. Acesso em: 08 out. 2006.

FREE CULTURE. Free Culture Manifest. 2006. Disponível em:

$<$ http://freeculture.org/manifest.php>. Acesso em: 22 dez. 2006.

FREE SOFTWARE FOUNDATION (FSF). About free software. Boston: FSF, 1999. Disponível em:

$<$ http://www.gnu.org/philosophy/philosophy. $\mathrm{html}$ \#AboutFreeSoftware>. Acesso em: 10 dez. 2005.

. GPLv3, 2nd discussion draft.

2006. Disponível em:

<http://gplv3.fsf.org/gpl-draft-2006-07-

27.html>. Acesso em: 18 dez. 2006.

HEXSEL, Roberto A. Software livre.

Curitiba, 2003. Disponível em:

<http://www.inf.ufpr.br/info/techrep/RT_DIN F004_2002.pdf>. Acesso em: 14 fev. 2005.

KUHN, Bradley M.; STALLMAN, Richard. Freedom or power. Boston, 2001.

Disponível em:

<http://www.gnu.or/philosophy/freedom-orpower.en.html>. Acesso em: 11 dez. 2005.
LEMOS, André. Cibercultura, cultura e identidade: em direção a uma "cultura copyleft"? Contemporânea, Salvador, v. 2, n. 2, p. 9-22, dez. 2004. Disponível em: $<$ http://www.contemporanea.poscom.ufba.b r/pdfdez04/ensaiociber.pdf $>$. Acesso em: 2 jan. 2007.

LESSIG, Lawrence. Free Culture: the nature and future of creativity. New York: Penguin Books, 2004.

MEFFE, Corinto; GUALBERTO, Marcio Alexandre $M$. Direito à comunicação na Sociedade da Informação: o papel estratégico do software livre. Tematec, Brasília, v. 9, n.18, p.1-4, mar./abr. 2005.

PEREIRA, André Luiz Greve. Copyright $x$ copyleft: uma análise marxista da indústria de software. Salvador, 2004. Disponível em:

$<$ http://www.adm.ufba.br/copyright_copyleft -uma_analise.pdf $>$. Acesso em: 30 nov. 2005.

SARAVIA, Diego. Ontologia de la libertad del conocimiento y del software libre: luchas y debates. 2005. Disponível em: $<$ http://docs.hipatia.info/ontologia/>. Acesso em: 3 jan. 2007.

SHWINGEL, Carla. O copyleft o desenvolvimento colaborativo como bases da cultura livre. Razón y Palabra, Zaragoza, n.53, Octubre/Noviembre 2006. Disponível em: <http://www.cem.itesm.mx/dacs/publicacio nes/logos/anteriores/n53/cshwingel.html>. Acesso em: 12 jan. 2007.

STALLMAN, Richard M. Linux P2P entrevista Richard Stallman. Entrevista concedida ao site Linux P2P, em 7 fev. 2006. Disponível em:

<http://www.linuxdailylog.com/2006/02/linu xp2p-entrevista-richard-stallman.html>.

Acesso em: 2 jan. 2007.

The GNU Manifest. Boston, 1993. Disponível em: <http://www.gnu.org/gnu/manifest.en.html>. Acesso em: 3 dez. 2005. 
STIX, Gary. Some rights reserved.

Scientific American, New York, v. 288, n.3, p.46, March. 2003. Disponível em:

$<$ http://search.ebscohost.com/login.aspx?di rect=true $\& \mathrm{db}=\mathrm{aph} \& A \mathrm{~N}=9071533 \&$ site $=$ eho st-live>. Acesso em: 2 jan. 2007.

TOURAINE, Alain. Sociologie de l'action. Paris: Seuil, 1965.

\section{Valdir Morigi \\ Professor Adjunto III do Departamento de Ci- ências da Informação (FABICO/UFRGS) Professor do Programa de Pós-Graduação em Comunicação e Informação Doutor em Sociologia (FFLCH/USP) valdir.morigi@ufrgs.br \\ Dirce Maria Santin Bacharel em Biblioteconomia (UFRGS) Bibliotecária (Confederação Interestadual das Cooperativas Ligadas ao SICREDI) dirsantin@yahoo.com.br}

\section{Title}

Some reflections concerning the value of Free Software Movement for new information movement's creation

\section{Abstract}

This article presents the Free Software as a social movement, analyzing its identity, its competitors and its social model. It reflects about the Movement's values and conflicts for the intellectual freedom achievement and its contribution for the creation of new information movements, as the Free Culture, the Creative Commons and the Open Access. It also explains the capability of the free software in mobilizing communities for the information and communication technologies' development process, the information access and the knowledge socialization questioning, taking part in an effective way and creating the necessary conditions for the transformation of values and of the social institutions in the Information Society.
}

\section{Keywords}

Free Software Movement; Free Culture; Creative Commons; Open Access; Intellectual freedom.

\section{Título}

Reflexiones sobre los valores del Movimiento Software Libre en la creación de nuevos movimientos informacionales

\section{Resumen}

Presenta el Software Libre como movimiento social, analizando su identidad, sus adversarios y su modelo social. Reflexiona sobre los valores y las luchas del Movimiento por la libertad intelectual y su contribución en la creación de nuevos movimientos informacionales, como la Cultura Libre, el Creative Commons y el Open Access. Diserta sobre la capacidad del Software Libre de movilizar y articular comunidades a cuestionaren el proceso de desenvolvimiento de las tecnologías de información y comunicación, las maneras de acceso a la información y la socialización del conocimiento, participando efectivamente y creando las condiciones necesarias a la transformación de los valores y de las instituciones sociales en la Sociedad de la Información.

\section{Palabras Clave}

Movimiento Software Libre; Cultura Libre; Creative Commons; Open Access; Libertad intelectual.

Recebido em: 21.03.2007

Aceito em: 08.04.2007 